QUARTERLY OF APPLIED MATHEMATICS

VOLUME LXVII, NUMBER 2

JUNE 2009, PAGES 249-263

S 0033-569X(09)01110-2

Article electronically published on March 19, 2009

\title{
ON THE STABILITY OF MINDLIN-TIMOSHENKO PLATES
}

BY

\author{
HUGO D. FERNÁNDEZ SARE
}

Department of Mathematics and Statistics, University of Konstanz, 78457, Konstanz, Germany

Abstract. We consider a Mindlin-Timoshenko model with frictional dissipations acting on the equations for the rotation angles. We prove that this system is not exponentially stable independent of any relations between the constants of the system, which is different from the analogous one-dimensional case. Moreover, we show that the solution decays polynomially to zero, with rates that can be improved depending on the regularity of the initial data.

1. Introduction. The conservative Mindlin-Timoshenko model in the two-dimensional case is given by

$$
\begin{aligned}
& \rho h w_{t t}-K\left[\frac{\partial}{\partial x}\left(\psi+\frac{\partial w}{\partial x}\right)+\frac{\partial}{\partial y}\left(\varphi+\frac{\partial w}{\partial y}\right)\right]=0 \\
& \frac{\rho h^{3}}{12} \psi_{t t}-D\left(\frac{\partial^{2} \psi}{\partial x^{2}}+\frac{1-\mu}{2} \frac{\partial^{2} \psi}{\partial y^{2}}+\frac{1+\mu}{2} \frac{\partial^{2} \varphi}{\partial x \partial y}\right)+K\left(\psi+\frac{\partial w}{\partial x}\right)=0, \\
& \frac{\rho h^{3}}{12} \varphi_{t t}-D\left(\frac{\partial^{2} \varphi}{\partial y^{2}}+\frac{1-\mu}{2} \frac{\partial^{2} \varphi}{\partial x^{2}}+\frac{1+\mu}{2} \frac{\partial^{2} \psi}{\partial x \partial y}\right)+K\left(\varphi+\frac{\partial w}{\partial y}\right)=0
\end{aligned}
$$

where $\Omega \subset \mathbb{R}^{2}$ is bounded, $\rho$ is the (constant) mass per unit of surface area, $h$ is the (uniform) plate thickness, $\mu$ is Poisson's ratio $\left(0<\mu<\frac{1}{2}\right.$ in physical situations), $D$ is the modulus of flexural rigidity, and $K$ is the shear modulus. The functions $w, \psi$ and $\varphi$ depend on $(t, x, y) \in[0, \infty) \times \Omega$, where $w$ models the transverse displacement of the plate, and $\psi, \varphi$ are the rotation angles of a filament of the plate; cp. [7, 8].

The main difference of this system to the analogous one-dimensional case $(\varphi \equiv 0)$ is that here another equation for rotation angles is considered. Note also that the coupling between the equations of the rotational angles $(\psi, \varphi)$ and the displacement equation $w$ is weaker than in one dimension. That is, while in two dimensions the coupling is given by the gradient of the functions (see system (1.8)-(1.9)), in the one-dimensional case

Received September 17, 2007.

2000 Mathematics Subject Classification. Primary 35B40, 74H40.

Key words and phrases. Timoshenko plates, non-exponential stability, polynomial stability.

The author was supported by the DFG-project "Hyperbolic Thermoelasticity" (RA 504/3-3).

E-mail address: hugosare@lncc.br 
the coupling is given by partial derivatives $\psi_{x}$ and $\varphi_{y}$ in (1.1), $w_{x}$ in (1.2), and $w_{y}$ in (1.3). Therefore the questions of how it is possible to stabilize the system and find "sufficient" dissipations to produce exponential stability are interesting, but they are not much studied in the literature.

In [7, Lagnese considered a bounded domain $\Omega$ having a Lipschitz boundary $\Gamma$ such that $\Gamma=\bar{\Gamma}_{0} \cup \bar{\Gamma}_{1}$, where $\Gamma_{0}$ and $\Gamma_{1}$ are relatively open, disjoints subsets of $\Gamma$ with $\Gamma_{1} \neq \emptyset$. He considered the following boundary conditions:

$$
\begin{aligned}
& w=\psi=\varphi=0 \quad \text { in } \Gamma_{0}, \\
& K\left(\frac{\partial w}{\partial x}+\psi, \frac{\partial w}{\partial y}+\varphi\right) \cdot \nu=m_{1} \text { in } \Gamma_{1}, \\
& D\left(\frac{\partial \psi}{\partial x}+\mu \frac{\partial \varphi}{\partial y}, \frac{1-\mu}{2}\left(\frac{\partial \varphi}{\partial x}+\frac{\partial \psi}{\partial y}\right)\right) \cdot \nu=m_{2} \text { in } \Gamma_{1}, \\
& D\left(\frac{1-\mu}{2}\left(\frac{\partial \varphi}{\partial x}+\frac{\partial \psi}{\partial y}\right), \frac{\partial \varphi}{\partial y}+\mu \frac{\partial \psi}{\partial x}\right) \cdot \nu=m_{3} \text { in } \Gamma_{1},
\end{aligned}
$$

where $\nu:=\left(\nu_{1}, \nu_{2}\right)$ is the unit exterior normal to $\Gamma=\partial \Omega$ and $\left\{m_{1}, m_{2}, m_{3}\right\}$ are the linear boundary feedbacks given by

$$
\left\{m_{1}, m_{2}, m_{3}\right\}=-F\left\{w_{t}, \psi_{t}, \varphi_{t}\right\},
$$

with $F=\left[f_{i j}\right]$ a $3 \times 3$ matrix of real $L^{\infty}\left(\Gamma_{1}\right)$ functions such that $F$ is symmetric and positive semidefinite on $\Gamma_{1}$. In that condition, Lagnese proved that the system (1.1)-(1.3) is exponentially stable, without any restrictions on the coefficients of the system. The same result is obtained by Muñoz Rivera and Portillo Oquendo [10, where, in (1.5)-(1.7), they consider boundary dissipations of memory type; that is,

$$
\begin{array}{r}
w+K \int_{0}^{t} g_{1}(t-s)\left(\frac{\partial w}{\partial x}(s)+\psi(s), \frac{\partial w}{\partial y}(s)+\varphi(s)\right) \cdot \nu d s=0 \text { in } \Gamma_{1}, \\
\psi+D \int_{0}^{t} g_{2}(t-s)\left(\frac{\partial \psi(s)}{\partial x}+\mu \frac{\partial \varphi(s)}{\partial y}, \frac{1-\mu}{2}\left(\frac{\partial \varphi(s)}{\partial x}+\frac{\partial \psi(s)}{\partial y}\right)\right) \cdot \nu d s=0 \quad \text { in } \Gamma_{1}, \\
\varphi+D \int_{0}^{t} g_{3}(t-s)\left(\frac{1-\mu}{2}\left(\frac{\partial \varphi(s)}{\partial x}+\frac{\partial \psi(s)}{\partial y}\right), \frac{\partial \varphi(s)}{\partial y}+\mu \frac{\partial \psi(s)}{\partial x}\right) \cdot \nu d s=0 \quad \text { in } \Gamma_{1} .
\end{array}
$$

With these boundary feedbacks, together with condition (1.4), they proved that the solutions of the system (1.1)-(1.3) are exponentially stable provided that the kernels have exponential behavior and are polynomially stable for kernels of polynomial type. Similar dissipations are used by De Lima Santos [3], where the author considered a Timoshenko model in $\Omega \subset \mathbb{R}^{n}$.

In this work, we are interested in introducing another type of dissipation. For example, taking into account the papers mentioned above, if we consider three frictional internal dissipations into the system; that is, introducing the terms $w_{t}$ in (1.1), $\psi_{t}$ in (1.2), and $\varphi_{t}$ in (1.3), the exponential behavior of the solutions of the system is easily obtained. The natural questions are the following: what happens if we remove one of these dissipations and, of course, which is the "natural" candidate to be removed? Looking at the onedimensional model we can deduce some conclusions in order to solve these questions. In 
[12, 14, the authors considered the one-dimensional damped Timoshenko system

$$
\begin{array}{ll}
\rho_{1} \varphi_{t t}-k\left(\varphi_{x}+\psi\right)_{x}=0 & \text { in }(0, L), \\
\rho_{2} \psi_{t t}-b \psi_{x x}+k\left(\varphi_{x}+\psi\right)+d \psi_{t}=0 & \text { in }(0, L),
\end{array}
$$

and proved that the solution of the system is exponentially stable if and only if the wave speeds of the system are equal, that is, if

$$
\frac{\rho_{1}}{k}=\frac{\rho_{2}}{b} .
$$

The Timoshenko model (1.8)-(1.9) with several types of dissipations has been studied by many authors; see for example [4, 5, 6, 11, 12, 14] and the references therein. The common point in almost every work is the condition (1.10), necessary and sufficient to obtain exponential stability. Note that, removing the dissipative term $d \psi_{t}$ in (1.9) and putting $\varphi_{t}$ in (1.8), we can deduce that the system is not exponentially stable independently if (1.10) holds or not. From these observations for the system (1.8)-(1.9) we can establish an equivalent problem in two dimensions. That is, introducing frictional dissipations in the rotational angle equations (1.2)-(1.3), we obtain a new dissipative system where the rate of decay for the solutions of that system appears as an open problem to be analyzed.

In other words, the purpose of this paper is to study the stability of the Timoshenko system

$$
\begin{aligned}
& \rho_{1} w_{t t}-K \operatorname{div}\left(\psi+\frac{\partial w}{\partial x}, \varphi+\frac{\partial w}{\partial y}\right)=0 \\
& \rho_{2} \psi_{t t}-D \operatorname{div}\left(\frac{\partial \psi}{\partial x}+\mu \frac{\partial \varphi}{\partial y}, \frac{1-\mu}{2}\left(\frac{\partial \varphi}{\partial x}+\frac{\partial \psi}{\partial y}\right)\right)+K\left[\psi+\frac{\partial w}{\partial x}\right]+d_{1} \psi_{t}=0, \\
& \rho_{2} \varphi_{t t}-D \operatorname{div}\left(\frac{1-\mu}{2}\left(\frac{\partial \varphi}{\partial x}+\frac{\partial \psi}{\partial y}\right), \frac{\partial \varphi}{\partial y}+\mu \frac{\partial \psi}{\partial x}\right)+K\left[\varphi+\frac{\partial w}{\partial y}\right]+d_{2} \varphi_{t}=0,
\end{aligned}
$$

where $\rho_{1}:=\rho h$, and $\rho_{2}:=\frac{\rho h^{3}}{12}$ in the system (1.1)-(1.3). We will prove that the system (1.11) - (1.13) is not exponentially stable, independent of any relation between the constants of the system. This leads to a different result from the one obtained in [12] in the one-dimensional case, where the condition (1.10) was sufficient and necessary to obtain exponential stability. Moreover, using multiplier techniques and Prüss et al.'s result [13, we will prove that the system (1.11)-(1.13) is polynomially stable with rates that can be improved depending on the initial data. We would like to add here that this is the first time that the asymptotic behavior of the system (1.11)-(1.13) has been studied, and that our analysis clearly shows the differences in the dimensions.

The paper is organized as follows: In Section 2 we shall look at the existence and uniqueness results using semigroup theory. The Timoshenko system (1.11)-(1.13) is shown to be not exponentially stable subject to mixed boundary conditions in Section 3 . Finally, in Section 4 we study the polynomial stability of the system (1.11)-(1.13) with Dirichlet boundary conditions.

2. Existence and uniqueness. We will use the standard notation $H^{k}(\Omega)$ or $H_{0}^{k}(\Omega)$ to denote usual Sobolev spaces of order $k$ over the regular domain $\Omega$, and set $L^{2}(\Omega)=$ 
$H^{0}(\Omega)$. We consider the Timoshenko system (1.11)-1.13) with the following Dirichlet boundary conditions:

$$
w(x, t)=\psi(x, t)=\varphi(x, t)=0 \text { in } \partial \Omega \times \mathbb{R}^{+},
$$

and initial conditions

$$
\begin{aligned}
& w(x, 0)=w_{0}(x), \quad w_{t}(x, 0)=w_{1}(x), \quad \text { in } \Omega, \\
& \psi(x, 0)=\psi_{0}(x), \quad \psi_{t}(x, 0)=\psi_{1}(x), \quad \text { in } \quad \Omega, \\
& \varphi(x, 0)=\varphi_{0}(x), \quad \varphi_{t}(x, 0)=\varphi_{1}(x), \quad \text { in } \quad \Omega .
\end{aligned}
$$

In order to obtain existence, uniqueness, and stability results, we will use semigroup theory. For this purpose we rewrite the system an as evolution equation for

$$
U=\left(w, w_{t}, \psi, \psi_{t}, \varphi, \varphi_{t}\right)^{\prime} \equiv\left(u^{1}, u^{2}, u^{3}, u^{4}, u^{5}, u^{6},\right)^{\prime} .
$$

Then $U$ formally satisfies

$$
U_{t}=\mathcal{A}_{1} U, \quad U(0)=U_{0},
$$

where $U_{0}=\left(w_{0}, w_{1}, \psi_{0}, \psi_{1}, \varphi_{0}, \varphi_{1}\right)^{\prime}$, and $\mathcal{A}_{1}$ is the (yet formal) differential operator

$$
\mathcal{A}_{1}:=\left(\begin{array}{cccccc}
0 & I d & 0 & 0 & 0 & 0 \\
\frac{K}{\rho_{1}} \Delta & 0 & \frac{K}{\rho_{1}} \partial_{x} & 0 & \frac{K}{\rho_{1}} \partial_{y} & 0 \\
0 & 0 & 0 & I d & 0 & 0 \\
-\frac{K}{\rho_{2}} \partial_{x} & 0 & \mathcal{B}_{1} & -\frac{d_{1}}{\rho_{2}} I d & \frac{D}{\rho_{2}}\left(\frac{1+\mu}{2}\right) \partial_{x y}^{2} & 0 \\
0 & 0 & 0 & 0 & 0 & I d \\
-\frac{K}{\rho_{2}} \partial_{y} & 0 & \frac{D}{\rho_{2}}\left(\frac{1+\mu}{2}\right) \partial_{x y}^{2} & 0 & \mathcal{B}_{2} & -\frac{d_{2}}{\rho_{2}} I d
\end{array}\right),
$$

where the differential operators $\mathcal{B}_{i}(i=1,2)$, are defined by

$$
\begin{aligned}
& \mathcal{B}_{1}=\frac{D}{\rho_{2}}\left[\partial_{x}^{2}+\left(\frac{1-\mu}{2}\right) \partial_{y}^{2}\right]-\frac{k}{\rho_{2}} I d, \\
& \mathcal{B}_{2}=\frac{D}{\rho_{2}}\left[\left(\frac{1-\mu}{2}\right) \partial_{x}^{2}+\partial_{y}^{2}\right]-\frac{k}{\rho_{2}} I d .
\end{aligned}
$$

Let

$$
\mathcal{H}_{1}:=H_{0}^{1}(\Omega) \times L^{2}(\Omega) \times H_{0}^{1}(\Omega) \times L^{2}(\Omega) \times H_{0}^{1}(\Omega) \times L^{2}(\Omega)
$$

be the Hilbert space. In order to endow the space $\mathcal{H}_{1}$ with a norm associated to the energy of the system (1.11)-(1.13), we will use the following result.

Lemma 2.1. There exists $\alpha_{0}>0$ such that, for all $(\psi, \varphi) \in\left[H_{0}^{1}(\Omega)\right]^{2}$,

$$
\begin{aligned}
\int_{\Omega}\left[\left|\psi_{x}\right|^{2}+\left|\varphi_{y}\right|^{2}+\right. & \left.\left(\frac{1-\mu}{2}\right)\left|\psi_{y}+\varphi_{x}\right|^{2}+\mu \psi_{x} \bar{\varphi}_{y}+\mu \varphi_{y} \bar{\psi}_{x}\right] d x d y \\
& \geq \alpha\left[\|\psi\|_{H^{1}}^{2}+\|\varphi\|_{H^{1}}^{2}\right] .
\end{aligned}
$$


Moreover, for every $K_{0}>0$ there exists $\beta:=\beta\left(K_{0}\right)>0$ such that for all $K \geq K_{0}$ and for all $(w, \psi, \varphi) \in\left[H_{0}^{1}(\Omega)\right]^{3}$,

$$
\begin{aligned}
& \int_{\Omega}\left[\left|\psi_{x}\right|^{2}+\left|\varphi_{y}\right|^{2}+\left(\frac{1-\mu}{2}\right)\left|\psi_{y}+\varphi_{x}\right|^{2}+\mu \psi_{x} \bar{\varphi}_{y}+\mu \varphi_{y} \bar{\psi}_{x}\right] d x d y \\
& \quad+K \int_{\Omega}\left|\psi+w_{x}\right|^{2} d x d y+K \int_{\Omega}\left|\varphi+w_{x}\right|^{2} d x d y \geq \beta\left[\|\nabla \psi\|_{L^{2}}^{2}+\|\nabla \varphi\|_{L^{2}}^{2}+\|\nabla w\|_{L^{2}}^{2}\right] .
\end{aligned}
$$

Proof. It is a direct consequence of Korn's Inequality; see [7].

Then, using the previous Lemma, we can obtain that

$$
\begin{aligned}
\|U\|_{\mathcal{H}_{1}}^{2}= & \left\|\left(u^{1}, u^{2}, u^{3}, u^{4}, u^{5}, u^{6}\right)\right\|_{\mathcal{H}_{1}}^{2} \\
= & \rho_{1}\left\|u^{2}\right\|_{L^{2}}^{2}+\rho_{2}\left\|u^{4}\right\|_{L^{2}}^{2}+\rho_{2}\left\|u^{6}\right\|_{L^{2}}^{2}+D\left\|u_{x}^{3}\right\|_{L^{2}}^{2}+D\left\|u_{y}^{5}\right\|_{L^{2}}^{2} \\
& +K\left\|u^{3}+u_{x}^{1}\right\|_{L^{2}}^{2}+K\left\|u^{5}+u_{y}^{1}\right\|_{L^{2}}^{2}+\mu\left(u_{x}^{3}, u_{y}^{5}\right)_{L^{2}}+\mu\left(u_{y}^{5}, u_{x}^{3}\right)_{L^{2}}
\end{aligned}
$$

is equivalent with the usual norm in $\mathcal{H}_{1}$.

Therefore, it is not difficult to prove that the operator $\mathcal{A}_{1}$ is maximal-dissipative; that is, $\mathcal{A}_{1}$ is the infinitesimal generator of a $C_{0}$ contraction semigroup on $\mathcal{H}_{1}$. Thus, we have the following result about existence and uniqueness of solutions.

Theorem 2.2. Let $U_{0}=\left(w_{0}, w_{1}, \psi_{0}, \psi_{1}, \varphi_{0}, \varphi_{1}\right)^{\prime} \in \mathcal{H}_{1}$. Then there exists a unique solution $U(t)=\left(w, w_{t}, \psi, \psi_{t}, \varphi, \varphi_{t}\right)^{\prime}$ to the system (1.11)-(1.13) with Dirichlet boundary conditions (2.1) satisfying

$$
U \in C\left(\mathbb{R}^{+} ; D\left(\mathcal{A}_{1}\right)\right) \cap C^{1}\left(\mathbb{R}^{+} ; \mathcal{H}_{1}\right) .
$$

Moreover, if $U_{0} \in D\left(\mathcal{A}_{1}^{n}\right)$, then

$$
U \in C^{n-k}\left(\mathbb{R}^{+} ; D\left(\mathcal{A}_{1}^{k}\right)\right), \quad k=0,1, \cdots, n .
$$

REMARK 2.3. The same analysis can be applied to obtain existence and uniqueness results for mixed boundary conditions.

3. Non-exponential stability. In this section we will prove that the system (1.11)(1.13) is not exponentially stable for suitable boundary conditions. In fact, we consider $\Omega \subset \mathbb{R}^{2}$ as the rectangle

$$
\Omega:=\left[0, L_{1}\right] \times\left[0, L_{2}\right], \quad \text { with } \quad L_{1}, L_{2}>0 .
$$

We define the sets

$$
\begin{aligned}
& \Gamma_{1}:=\left\{(x, y): 0<x<L_{1}, y=0, L_{2}\right\} \\
& \Gamma_{2}:=\left\{(x, y): 0<y<L_{2}, x=0, L_{1}\right\} .
\end{aligned}
$$


Note that $\Gamma:=\partial \Omega=\bar{\Gamma}_{1} \cup \bar{\Gamma}_{2}$. The boundary conditions considered for the system (1.11) -(1.13) are the following:

$$
\begin{array}{lll}
w=0 & \text { in } \Gamma, \\
\psi=0, & & \left(\frac{1-\mu}{2}\left(\frac{\partial \varphi}{\partial x}+\frac{\partial \psi}{\partial y}\right), \frac{\partial \varphi}{\partial y}+\mu \frac{\partial \psi}{\partial x}\right) \cdot \nu=0 \quad \text { in } \Gamma_{1}, \\
\varphi=0, & & \left(\frac{\partial \psi}{\partial x}+\mu \frac{\partial \varphi}{\partial y}, \frac{1-\mu}{2}\left(\frac{\partial \varphi}{\partial x}+\frac{\partial \psi}{\partial y}\right)\right) \cdot \nu=0 \quad \text { in } \Gamma_{2},
\end{array}
$$

where $\nu:=\left(\nu_{1}, \nu_{2}\right)$ is the unit exterior normal to $\Gamma=\partial \Omega$. Therefore, the semigroup formulation is given in the Hilbert space,

$$
\mathcal{H}_{2}:=H_{0}^{1}(\Omega) \times L^{2}(\Omega) \times H_{\Gamma_{1}}^{1}(\Omega) \times L^{2}(\Omega) \times H_{\Gamma_{2}}^{1}(\Omega) \times L^{2}(\Omega),
$$

where

$$
H_{\Gamma_{i}}^{1}(\Omega):=\left\{u \in H^{1}(\Omega): u=0 \text { in } \Gamma_{i}\right\} \quad(i=1,2)
$$

and with the same norm given by (2.4).

We shall use the following well-known result from semigroup theory (see, e.g., [9, Theorem 1.3.2]).

LEMma 3.1. A semigroup of contractions $\left\{\mathrm{e}^{t \mathcal{A}}\right\}_{t \geq 0}$ in a Hilbert space with norm $\|\cdot\|$ is exponentially stable if and only if

(i) the resolvent set $\varrho(\mathcal{A})$ of $\mathcal{A}$ contains the imaginary axis

and

hold.

$$
\text { (ii) } \limsup _{\lambda \rightarrow \pm \infty}\left\|(i \lambda I d-\mathcal{A})^{-1}\right\|<\infty
$$

Hence it suffices to show the existence of sequences $\left(\lambda_{n}\right)_{n} \subset \mathbb{R}$ with

$$
\lim _{n \rightarrow \infty}\left|\lambda_{n}\right|=\infty,
$$

and $\left(U_{n}\right)_{n} \subset D\left(\mathcal{A}_{1}\right),\left(F_{n}\right)_{n} \subset \mathcal{H}$, such that

$$
\left(i \lambda_{n} I d-\mathcal{A}_{1}\right) U_{n}=F_{n} \quad \text { is bounded and } \lim _{n \rightarrow \infty}\left\|U_{n}\right\|_{\mathcal{H}_{1}}=\infty .
$$

As $F_{n} \equiv F$ we choose $F:=\left(0, f^{2}, 0, f^{4}, 0, f^{6}\right)^{\prime}$ with

$$
\begin{array}{lll}
f^{2}:=F^{2} \sin \left(\delta \lambda_{1} x\right) \sin \left(\delta \lambda_{2} y\right), & F^{2} \neq 0(\text { constant }), \\
f^{4}:=F^{4} \cos \left(\delta \lambda_{1} x\right) \sin \left(\delta \lambda_{2} y\right), & F^{4} \neq 0(\text { constant }), \\
f^{6}:=F^{6} \sin \left(\delta \lambda_{1} x\right) \cos \left(\delta \lambda_{2} y\right), & F^{6} \neq 0(\text { constant }),
\end{array}
$$

where

$$
\lambda_{1} \equiv \lambda_{1, n}:=\frac{n \pi}{\delta L_{1}}, \quad \lambda_{2} \equiv \lambda_{2, n}:=\frac{n \pi}{\delta L_{2}} \quad(n \in \mathbb{N}), \quad \delta:=\sqrt{\frac{\rho_{1}}{k}} .
$$

Finally we define

$$
\lambda \equiv \lambda_{n}:=\sqrt{\lambda_{1}^{2}+\lambda_{2}^{2}}
$$

The solution $U=\left(v^{1}, v^{2}, v^{3}, v^{4}, v^{5}, v^{6}\right)^{\prime}$ of the resolvent equation

$$
\left(i \lambda I d-\mathcal{A}_{1}\right) U=F
$$


should satisfy

$$
\begin{aligned}
i \lambda v^{1}-v^{2} & =0, \\
i \lambda v^{2}-\frac{k}{\rho_{1}}\left(v^{3}+v_{x}^{1}\right)_{x}--\frac{k}{\rho_{1}}\left(v^{5}+v_{y}^{1}\right)_{y} & =f^{2}, \\
i \lambda v^{3}-v^{4} & =0, \\
i \lambda v^{4}-\frac{D}{\rho_{2}}\left[v_{x x}^{3}+\left(\frac{1-\mu}{2}\right) v_{y y}^{3}+\left(\frac{1+\mu}{2}\right) v_{x y}^{5}\right]+\frac{k}{\rho_{2}}\left(v^{3}+v_{x}^{1}\right)+\frac{d_{1}}{\rho_{2}} v^{4} & =f^{4}, \\
i \lambda v^{5}-v^{6} & =0, \\
i \lambda v^{6}-\frac{D}{\rho_{2}}\left[\left(\frac{1-\mu}{2}\right) v_{x x}^{5}+v_{y y}^{5}+\left(\frac{1+\mu}{2}\right) v_{x y}^{3}\right]+\frac{k}{\rho_{2}}\left(v^{5}+v_{y}^{1}\right)+\frac{d_{2}}{\rho_{2}} v^{6} & =f^{6} .
\end{aligned}
$$

Eliminating $v^{2}, v^{4}, v^{6}$ we obtain the following system for $v^{1}, v^{3}, v^{5}$ :

$$
\begin{aligned}
-\lambda^{2} \rho_{1} v^{1}-k\left(v^{3}+v_{x}^{1}\right)_{x}-k\left(v^{5}+v_{y}^{1}\right)_{y} & =\rho_{1} f^{2}, \\
-\lambda^{2} \rho_{2} v^{3}-D\left[v_{x x}^{3}+\left(\frac{1-\mu}{2}\right) v_{y y}^{3}+\left(\frac{1+\mu}{2}\right) v_{x y}^{5}\right]+k\left(v^{3}+v_{x}^{1}\right)+i \lambda d_{1} v^{3} & =\rho_{2} f^{4}, \\
-\lambda^{2} \rho_{2} v^{5}-D\left[\left(\frac{1-\mu}{2}\right) v_{x x}^{5}+v_{y y}^{5}+\left(\frac{1+\mu}{2}\right) v_{x y}^{3}\right]+k\left(v^{5}+v_{y}^{1}\right)+i \lambda d_{2} v^{5} & =\rho_{2} f^{6} .
\end{aligned}
$$

System (3.4) can be solved by

$$
\begin{aligned}
v^{1}(x, y) & :=A \sin \left(\delta \lambda_{1} x\right) \sin \left(\delta \lambda_{2} y\right), \\
v^{3}(x, y) & :=B \cos \left(\delta \lambda_{1} x\right) \sin \left(\delta \lambda_{2} y\right), \\
v^{5}(x, y) & :=C \sin \left(\delta \lambda_{1} x\right) \cos \left(\delta \lambda_{2} y\right),
\end{aligned}
$$

where $A, B, C$ depend on $\lambda$ and will be determined explicitly in the sequel. Note that this choice is just compatible with the boundary conditions (3.1). System (3.4) is equivalent to finding $A, B, C$ such that

$$
\begin{aligned}
-\lambda^{2} \rho_{1} A+k \delta^{2}\left(\lambda_{1}^{2}+\lambda_{2}^{2}\right) A+k \delta \lambda_{1} B+k \delta \lambda_{2} C & =\rho_{1} F^{2}, \\
-\lambda^{2} \rho_{2} B+D \delta^{2} \lambda_{1}^{2} B+D\left(\frac{1-\mu}{2}\right) \delta^{2} \lambda_{2}^{2} B+D\left(\frac{1+\mu}{2}\right) \delta^{2} \lambda_{1} \lambda_{2} C & \\
+k B+k \delta \lambda_{1} A+i \lambda d_{1} B & =\rho_{2} F^{4}, \\
-\lambda^{2} \rho_{2} C+D\left(\frac{1-\mu}{2}\right) \delta^{2} \lambda_{1}^{2} C+D \delta^{2} \lambda_{2}^{2} C+D\left(\frac{1+\mu}{2}\right) \delta^{2} \lambda_{1} \lambda_{2} B & \\
+k C+k \delta \lambda_{2} A+i \lambda d_{2} C & =\rho_{2} F^{6} .
\end{aligned}
$$

Using the definitions of $\delta$ and $\lambda$, we obtain from (3.5) that

$$
B=-\frac{\lambda_{2}}{\lambda_{1}} C+\frac{1}{\lambda_{1}} \delta F^{2}
$$

or

$$
C=-\frac{\lambda_{1}}{\lambda_{2}} B+\frac{1}{\lambda_{2}} \delta F^{2}
$$


Using (3.9) in (3.6) we obtain

$$
\left[-\lambda^{2}\left(\rho_{2}-D \delta^{2}\left(\frac{1-\mu}{2}\right)\right)+k+i \lambda d_{1}\right] B+k \delta \lambda_{1} A=\rho_{2} F^{4}-D\left(\frac{1+\mu}{2}\right) \delta^{3} \lambda_{1} F^{2} .
$$

Similarly, using (3.8) in (3.7) we obtain

$$
\left[-\lambda^{2}\left(\rho_{2}-D \delta^{2}\left(\frac{1-\mu}{2}\right)\right)+k+i \lambda d_{2}\right] C+k \delta \lambda_{2} A=\rho_{2} F^{6}-D\left(\frac{1+\mu}{2}\right) \delta^{3} \lambda_{2} F^{2} .
$$

Let

$$
\Theta:=\rho_{2}-D \frac{\rho_{1}}{k}\left(\frac{1-\mu}{2}\right)
$$

Then, using the definition of $\delta$, we obtain from (3.10)-3.11 that $A, B$ satisfies

$$
\begin{aligned}
\left(-\lambda^{2} \Theta+k+i \lambda d_{1}\right) B+k \delta \lambda_{1} A & =\rho_{2} F^{4}-D\left(\frac{1+\mu}{2}\right) \delta^{3} \lambda_{1} F^{2}, \\
\left(-\lambda^{2} \Theta+k+i \lambda d_{2}\right) C+k \delta \lambda_{2} A & =\rho_{2} F^{6}-D\left(\frac{1+\mu}{2}\right) \delta^{3} \lambda_{2} F^{2} .
\end{aligned}
$$

REMARK 3.2. Note that the condition $\Theta=0$ in (3.12) gives a relationship (in the 2-dimensional case) similar to the relation

$$
\frac{\rho_{1}}{k}=\frac{\rho_{2}}{b},
$$

which is a necessary and sufficient condition for exponential stability in the 1-dimensional case; see [12. We will show that the system (1.11)-1.13) is non-exponentially stable and independent of any relation between the coefficients of the system, in particular of $\Theta=0$ in 3.12 .

Using (3.9) in (3.14) we obtain

$$
\begin{aligned}
\left(-\lambda^{2} \Theta+k+i \lambda d_{2}\right) \frac{\lambda_{1}^{2}}{\lambda_{2}^{2}} B-k \delta \lambda_{1} A= & -\frac{\lambda_{1}}{\lambda_{2}} \rho_{2} F^{6}+D\left(\frac{1+\mu}{2}\right) \delta^{3} \lambda_{1} F^{2} \\
& +\left(-\lambda^{2} \Theta+k+i \lambda d_{2}\right) \delta \lambda_{1} F^{2} .
\end{aligned}
$$

Then, adding the equalities (3.13) and (3.15) yields

$$
\begin{aligned}
{\left[\left(-\lambda^{2} \Theta+k+i \lambda d_{1}\right)+\left(-\lambda^{2} \Theta+k+i \lambda d_{2}\right) \frac{\lambda_{1}^{2}}{\lambda_{2}^{2}}\right] B=} & \rho_{2} F^{4}-\frac{\lambda_{1}}{\lambda_{2}} \rho_{2} F^{6} \\
& +\left(-\lambda^{2} \Theta+k+i \lambda d_{2}\right) \delta \lambda_{1} F^{2} .
\end{aligned}
$$

This is

$$
B=\frac{\rho_{2} F^{4}-\frac{\lambda_{1}}{\lambda_{2}} \rho_{2} F^{6}+\left(-\lambda^{2} \Theta+k+i \lambda d_{2}\right) \delta \lambda_{1} F^{2}}{-\lambda^{2} \Theta\left(1+\frac{\lambda_{1}^{2}}{\lambda_{2}^{2}}\right)+k\left(1+\frac{\lambda_{1}^{2}}{\lambda_{2}^{2}}\right)+i \lambda\left(d_{1}+d_{2} \frac{\lambda_{1}^{2}}{\lambda_{2}^{2}}\right)}
$$

and using (3.13) we have

$$
A=\frac{\rho_{2}}{k \delta \lambda_{1}} F^{4}-D\left(\frac{1+\mu}{2}\right) \frac{\delta^{2}}{k} F^{2}-\left(-\lambda^{2} \Theta+k+i \lambda d_{1}\right) B
$$


with $B$ given by (3.16). We define

$$
Q(\lambda):=-\lambda^{2} \Theta\left(1+\frac{\lambda_{1}^{2}}{\lambda_{2}^{2}}\right)+k\left(1+\frac{\lambda_{1}^{2}}{\lambda_{2}^{2}}\right)+i \lambda\left(d_{1}+d_{2} \frac{\lambda_{1}^{2}}{\lambda_{2}^{2}}\right),
$$

where, using the definitions of $\lambda_{i}(i=1,2)$, we can conclude that

$$
L:=\frac{\lambda_{1}}{\lambda_{2}}=\frac{L_{2}}{L_{1}}>0 .
$$

Therefore $Q(\lambda)$ is given by

$$
Q(\lambda)=-\lambda^{2} \Theta\left(1+L^{2}\right)+k\left(1+L^{2}\right)+i \lambda\left(d_{1}+d_{2} L^{2}\right) .
$$

We also define the following functions:

$$
\begin{aligned}
A_{1}(\lambda):= & \frac{\rho_{2}}{k \delta \lambda_{1}} F^{4}+\frac{1}{Q(\lambda)}\left[k \rho_{2}\left(L F^{6}-F^{4}\right)-D\left(\frac{1+\mu}{2}\right) \delta^{2}\left(1+L^{2}\right)\right] \\
A_{2}(\lambda):= & \frac{1}{Q(\lambda)}\left[-\lambda^{4} \lambda_{1} \Theta^{2} \delta F^{2}+i \lambda^{3} \lambda_{1} \Theta\left(d_{1}-d_{2}\right) \delta F^{2}+\lambda^{2} \lambda_{1}\left(d_{2} d_{1}+2 \Theta k\right) \delta F^{2}\right. \\
& +\lambda^{2} \Theta\left(\left(F^{4}-L F^{6}\right) \rho_{2}+D\left(\frac{1+\mu}{2}\right) \frac{\delta^{2}}{k}\left(1+L^{2}\right)\right)-i \lambda \lambda_{1}\left(d_{1}+d_{2}\right) k \delta F^{2} \\
& \left.+i \lambda\left(d_{1} \rho_{2}\left(L F^{6}-F^{4}\right)-D\left(\frac{1+\mu}{2}\right)\left(d_{1}+d_{2} L^{2}\right)\right)-\delta \lambda_{1} k F^{2}\right] .
\end{aligned}
$$

Then we have in (3.17) that

$$
A=A_{1}(\lambda)+A_{2}(\lambda)
$$

Recalling that

$$
v^{2}=i \lambda v^{1}=i \lambda A \sin \left(\delta \lambda_{1} x\right) \sin \left(\delta \lambda_{2} y\right)
$$

we get

$$
v^{2}=\left(i \lambda A_{1}(\lambda)+i \lambda A_{2}(\lambda)\right) \sin \left(\delta \lambda_{1} x\right) \sin \left(\delta \lambda_{2} y\right)
$$

Note that

$$
\begin{aligned}
\left\|U_{n}\right\|_{\mathcal{H}} & \geq\left\|v^{2}\right\|_{L^{2}} \\
& =\left(\int_{\Omega}\left|v^{2}\right|^{2} d x d y\right)^{1 / 2} \\
& \geq-C_{1}\left|\lambda A_{1}(\lambda)\right|+C_{2}\left|\lambda A_{2}(\lambda)\right|,
\end{aligned}
$$

where $C_{i}:=C_{i}\left(L_{1}, L_{2}\right)>0, i=1,2$. Then, to complete our result, it is sufficient to show that

(i) The sequence $\left\{\lambda A_{1}(\lambda)\right\}_{\lambda} \subset \mathbb{R}^{+}$is bounded, and

(ii) $\left|\lambda A_{2}(\lambda)\right| \rightarrow \infty$ as $\lambda \rightarrow \infty$, independent of any relation between the constants of the system. 
In fact, using the definitions of $\lambda, \lambda_{1}$ in (3.20) we obtain

$$
\lambda A_{1}(\lambda)=\frac{\rho_{2}}{k \delta} \sqrt{1+\frac{1}{L^{2}}} F^{4}+\frac{k \rho_{2}\left(L F^{6}-F^{4}\right)-D\left(\frac{1+\mu}{2}\right) \delta^{2}\left(1+L^{2}\right)}{-\lambda \Theta\left(1+L^{2}\right)+\frac{k}{\lambda}\left(1+L^{2}\right)+i\left(d_{1}+d_{2} L^{2}\right)} .
$$

Then $\left\{\lambda A_{1}(\lambda)\right\}_{\lambda}$ is bounded, which completes the proof of item (i). On the other hand, note that item (ii) is obvious in the case $\Theta \neq 0$. When $\Theta=0$ we have in (3.21) that

$$
\begin{aligned}
\lambda A_{2}(\lambda)= & \frac{1}{Q_{0}(\lambda)}\left[2 \lambda^{3} \lambda_{1} d_{2} d_{1} \delta F^{2}-i \lambda^{2} \lambda_{1}\left(d_{1}+d_{2}\right) k \delta F^{2}\right. \\
& \left.+i \lambda^{2}\left(d_{1} \rho_{2}\left(L F^{6}-F^{4}\right)-D\left(\frac{1+\mu}{2}\right)\left(d_{1}+d_{2} L^{2}\right)\right)-\delta \lambda \lambda_{1} k F^{2}\right],
\end{aligned}
$$

with

$$
Q_{0}(\lambda)=k\left(1+L^{2}\right)+i \lambda\left(d_{1}+d_{2} L^{2}\right) .
$$

Therefore $\left|\lambda A_{2}(\lambda)\right| \longrightarrow \infty$. Thus we have proved the following theorem.

Theorem 3.3. The Timoshenko system (1.11)-(1.13) with boundary conditions (3.1) is not exponentially stable, independent of any relation between the constants of the system.

REMARK 3.4. As in the 1-dimensional case, the non-exponential stability in Dirichlet boundary conditions (2.1) is still an open problem. Note also that the function that generates the non-exponential stability, that is $\left\{\lambda A_{2}(\lambda)\right\}_{\lambda}$, has the behavior as $\left|\lambda A_{2}(\lambda)\right| \sim$ $\circ\left(\lambda^{3}\right)$, which produces the expectation that, to show polynomial stability results, we will need energies of higher order; see Section 4.

4. Polynomial stability. In this section we shall prove that the system (1.11)(1.13) with boundary conditions (2.1) is polynomially stable. The energy of first order associated to the system (1.11) -(1.13) is given by

$$
\begin{aligned}
E_{1}(t) & :=E_{1}(t ; w, \psi, \varphi) \\
=\frac{1}{2} \int_{\Omega}\left[\rho_{1}\left|w_{t}\right|^{2}+\rho_{2}\left|\psi_{t}\right|^{2}+\rho_{2}\left|\varphi_{t}\right|^{2}+K\left|\psi+w_{x}\right|^{2}+K\left|\varphi+w_{x}\right|^{2}+D\left|\psi_{x}\right|^{2}\right. & \\
& \left.+D\left|\varphi_{y}\right|^{2}+\left(\frac{1-\mu}{2}\right) D\left|\psi_{y}+\varphi_{x}\right|^{2}+2 D \mu \psi_{x} \varphi_{y}\right] d x d y,
\end{aligned}
$$

which is obtained multiplying equation (1.11) by $w_{t}$, (1.12) by $\psi_{t}$, and (1.13) by $\varphi_{t}$. Also, we can define the energies

$$
E_{i+1}(t):=E_{1}\left(t ; \partial_{t}^{(i)} w, \partial_{t}^{(i)} \psi, \partial_{t}^{(i)} \varphi\right), \quad i=1,2,3 .
$$

It is not difficult to show that

$$
\frac{d}{d t} E_{i}(t)=-d_{1} \int_{\Omega}\left|\partial_{t}^{(i)} \psi\right|^{2} d x d y-d_{2} \int_{\Omega}\left|\partial_{t}^{(i)} \varphi\right|^{2} d x d y, \quad i=1,2,3,4 .
$$


We define

$$
F_{1}(t):=\int_{\Omega}\left[\rho_{1} w_{t} w+\rho_{2} \psi_{t} \psi+\rho_{2} \varphi_{t} \varphi+\frac{d_{1}}{2} \psi^{2}+\frac{d_{2}}{2} \varphi^{2}\right] d x d y .
$$

Then, multiplying equation (1.11) by $w,(1.12)$ by $\psi$, and (1.13) by $\varphi$, results in

$$
\begin{aligned}
\frac{d}{d t} F_{1}(t)= & -D \int_{\Omega}\left[\left|\psi_{x}\right|^{2}+\left|\varphi_{y}\right|^{2}+\left(\frac{1-\mu}{2}\right)\left|\psi_{y}+\varphi_{x}\right|^{2}+2 \mu \psi_{y} \varphi_{x}\right] d x d y \\
& -K \int_{\Omega}\left|\psi+w_{x}\right|^{2} d x d y-K \int_{\Omega}\left|\varphi+w_{x}\right|^{2} d x d y+\rho_{2} \int_{\Omega}\left|\psi_{t}\right|^{2} d x d y \\
& +\rho_{2} \int_{\Omega}\left|\varphi_{t}\right|^{2} d x d y+\rho_{1} \int_{\Omega}\left|w_{t}\right|^{2} d x d y .
\end{aligned}
$$

Let $q: \Omega \rightarrow \mathbb{R}$ defined by $q(x, y)=x$. We define

$$
F_{2}(t):=-D \int_{\Omega}\left(\psi_{x t}+\mu \varphi_{y t},\left(\frac{1-\mu}{2}\right)\left(\psi_{y t}-\varphi_{x t}\right)\right) \cdot \nabla w q(x, y) d x d y
$$

Then, differentiating equation (1.12) with respect to $t$ and multiplying by $q(x, y) w_{t}$ in $L^{2}(\Omega)$ results in

$$
\begin{aligned}
\frac{d}{d t} F_{2}(t)= & -K \int_{\Omega}\left|w_{t}\right|^{2} d x d y+\rho_{2} \int_{\Omega} \psi_{t t t} q(x, y) w_{t} d x d y+K \int_{\Omega} \psi_{t} q(x, y) w_{t} d x d y \\
& +d_{1} \int_{\Omega} \psi_{t t} q(x, y) w_{t} d x d y+D \int_{\Omega}\left(\psi_{x t}+\mu \varphi_{y t}\right) w_{t} d x d y \\
& -D \int_{\Omega}\left(\psi_{x t t}+\mu \varphi_{y t t},\left(\frac{1-\mu}{2}\right)\left(\psi_{y t t}-\varphi_{x t t}\right)\right) \cdot \nabla w q(x, y) d x d y
\end{aligned}
$$

where we can conclude that there exists

$$
C_{i}:=C_{i}\left(\rho_{1}, \rho_{2}, K, D, \mu, \Omega\right)>0, \quad i=1,2,
$$

such that

$$
\begin{aligned}
\frac{d}{d t} F_{2}(t) \leq & -\frac{K}{2} \int_{\Omega}\left|w_{t}\right|^{2} d x d y+C_{1}\left[|| \psi_{t}\left\|_{H^{1}}^{2}+\right\| \varphi_{t} \|_{H^{1}}^{2}\right]+C_{2} \int_{\Omega}\left[\left|\psi_{t t}\right|^{2}+\left|\psi_{t t t}\right|^{2}\right] d x d y \\
& -D \int_{\Omega}\left(\psi_{x t t}+\mu \varphi_{y t t},\left(\frac{1-\mu}{2}\right)\left(\psi_{y t t}-\varphi_{x t t}\right)\right) \cdot \nabla w q(x, y) d x d y .
\end{aligned}
$$

We will use the letter $C$ to denote several positive constants defined as in (4.7). Defining

$$
F_{3}(t):=F_{1}(t)+\frac{4 \rho_{1}}{K} F_{2}(t)
$$

and using (4.5) and (4.8) we have

$$
\begin{aligned}
\frac{d}{d t} F_{3}(t) & \leq-2 E_{1}(t)+C\left[\left\|\psi_{t}\right\|_{H^{1}}^{2}+|| \varphi_{t} \|_{H^{1}}^{2}\right]+C \int_{\Omega}\left[\left|\psi_{t t}\right|^{2}+\left|\psi_{t t t}\right|^{2}\right] d x d y \\
& -\frac{4 \rho_{1} D}{K} \int_{\Omega}\left(\psi_{x t t}+\mu \varphi_{y t t},\left(\frac{1-\mu}{2}\right)\left(\psi_{y t t}-\varphi_{x t t}\right)\right) \cdot \nabla w q(x, y) d x d y .
\end{aligned}
$$


REMARK 4.1. In (4.10) we already have the first order energy with negative signs, but it is necessary to estimate other higher-order terms. The following functions will be defined in order to estimate these terms.

First, note that using Korn's Inequality [2], we have that there exist constants $\alpha, \beta>0$ such that (see 7])

$$
\int_{\Omega}\left[\left|\psi_{x}\right|^{2}+\left|\varphi_{y}\right|^{2}+\left(\frac{1-\mu}{2}\right)\left|\psi_{y}+\varphi_{x}\right|^{2}+2 \mu \psi_{x} \varphi_{y}\right] d x d y \geq \alpha\left[\|\psi\|_{H^{1}}^{2}+\|\varphi\|_{H^{1}}^{2}\right]
$$

and

$$
\begin{gathered}
\int_{\Omega}\left[\left|\psi_{x}\right|^{2}+\left|\varphi_{y}\right|^{2}+\left(\frac{1-\mu}{2}\right)\left|\psi_{y}+\varphi_{x}\right|^{2}+2 \mu \psi_{x} \varphi_{y}\right] d x d y \\
+K \int_{\Omega}\left|\psi+w_{x}\right|^{2} d x d y+K \int_{\Omega}\left|\varphi+w_{x}\right|^{2} d x d y \geq \beta\left[\|\nabla \psi\|_{L^{2}}^{2}+\|\nabla \varphi\|_{L^{2}}^{2}+\|\nabla w\|_{L^{2}}^{2}\right] .
\end{gathered}
$$

On the other hand, differentiating equations (1.12)-(1.13) with respect to $t$ and multiplying by $\psi_{t}$ and $\varphi_{t}$ respectively, results in

$$
\begin{aligned}
& \frac{d}{d t} \int_{\Omega} \rho_{2}\left[\psi_{t t} \psi_{t}+\varphi_{t t} \varphi_{t}\right] d x d y \\
& =-D \int_{\Omega}\left[\left|\psi_{x t}\right|^{2}+\left|\varphi_{y t}\right|^{2}+\left(\frac{1-\mu}{2}\right)\left|\psi_{y t}+\varphi_{x t}\right|^{2}+2 \mu \psi_{y t} \varphi_{x t}\right] d x d y \\
& \quad+\rho_{2} \int_{\Omega}\left|\psi_{t t}\right|^{2} d x d y-K \int_{\Omega}\left(\psi_{t}+w_{x t}\right) \psi_{t} d x d y-d_{1} \int_{\Omega} \psi_{t t} \psi_{t} d x d y \\
& \quad+\rho_{2} \int_{\Omega}\left|\varphi_{t t}\right|^{2} d x d y-K \int_{\Omega}\left(\varphi_{t}+w_{x t}\right) \varphi_{t} d x d y-d_{2} \int_{\Omega} \varphi_{t t} \varphi_{t} d x d y
\end{aligned}
$$

Then, defining

$$
F_{4}(t):=\int_{\Omega}\left[\rho_{2} \psi_{t t} \psi_{t}+\rho_{2} \varphi_{t t} \varphi_{t}+K \nabla w \cdot\left(\psi_{t}, \varphi_{t}\right)\right] d x d y
$$

and using (4.11) we obtain

$$
\begin{aligned}
\frac{d}{d t} F_{4}(t) \leq & -D \alpha\left[\left\|\psi_{t}\right\|_{H^{1}}^{2}+\left\|\varphi_{t}\right\|_{H^{1}}^{2}\right]+C \int_{\Omega}\left[\left|\psi_{t t}\right|^{2}+\left|\varphi_{t t}\right|^{2}\right] d x d y \\
& +K \int_{\Omega}\left(w_{x} \psi_{t t}+w_{y} \varphi_{t t}\right) d x d y
\end{aligned}
$$

Let

$$
F_{5}(t):=F_{3}(t)+\frac{C}{D \alpha} F_{4}(t) .
$$

Then, from (4.10) and (4.15) we have

$$
\begin{aligned}
\frac{d}{d t} F_{5}(t) \leq & -2 E_{1}(t)+C \int_{\Omega}\left[\left|\psi_{t t}\right|^{2}+\left|\varphi_{t t}\right|^{2}+\left|\psi_{t t t}\right|^{2}\right] d x d y+\frac{C K}{D \alpha} \int_{\Omega}\left(w_{x} \psi_{t t}+w_{y} \varphi_{t t}\right) d x d y \\
& -\frac{4 \rho_{1} D}{K} \int_{\Omega}\left(\psi_{x t t}+\mu \varphi_{y t t},\left(\frac{1-\mu}{2}\right)\left(\psi_{y t t}-\varphi_{x t t}\right)\right) \cdot \nabla w q(x, y) d x d y .
\end{aligned}
$$


Note that, using the definition of $E_{1}(t)$ and the inequality (4.12), we obtain

$$
-2 E_{1}(t) \leq-E_{1}(t)-\frac{\beta}{2}\|\nabla w\|_{L^{2}}^{2} .
$$

Therefore, applying (4.18) in (4.17) we can deduce that

$$
\begin{aligned}
\frac{d}{d t} F_{5}(t) \leq & -E_{1}(t)-\frac{\beta}{4}\|\nabla w\|_{L^{2}}^{2}+C \int_{\Omega}\left|\psi_{t t t}\right|^{2} d x d y \\
& +C_{\beta}\left[\left\|\psi_{t t}\right\|_{H^{1}}^{2}+\left\|\varphi_{t t}\right\|_{H^{1}}^{2}\right]
\end{aligned}
$$

where $C_{\beta}>0$ is defined as in (4.7) and depends also on $\beta>0$.

Similarly as in (4.13), differentiating equations (1.12)-(1.13) with respect to $t$ two times, and multiplying by $\psi_{t t}$ and $\varphi_{t t}$ respectively, we can deduce

$$
\begin{aligned}
& \frac{d}{d t} \int_{\Omega} \rho_{2}\left[\psi_{t t t} \psi_{t t}+\varphi_{t t t} \varphi_{t t}\right] d x d y \\
& \quad \leq-D \alpha\left[\left\|\psi_{t t}\right\|_{H^{1}}^{2}+\left\|\varphi_{t t}\right\|_{H^{1}}^{2}\right]+\rho_{2} \int_{\Omega}\left[\left|\psi_{t t t}\right|^{2}+\left|\varphi_{t t t}\right|^{2}\right] d x d y \\
& \quad-K \int_{\Omega}\left(\psi_{t t}+w_{x t t}\right) \psi_{t t} d x d y-d_{1} \int_{\Omega} \psi_{t t t} \psi_{t t} d x d y \\
& \quad-K \int_{\Omega}\left(\varphi_{t t}+w_{x t t}\right) \varphi_{t t} d x d y-d_{2} \int_{\Omega} \varphi_{t t t} \varphi_{t t} d x d y
\end{aligned}
$$

where inequality (4.11) is used. We define

$$
F_{6}(t):=\int_{\Omega}\left[\rho_{2} \psi_{t t t} \psi_{t t}+\rho_{2} \varphi_{t t t} \varphi_{t t}-K \nabla w_{t} \cdot\left(\psi_{t t}, \varphi_{t t}\right)+K \nabla w \cdot\left(\psi_{t t t}, \varphi_{t t t}\right)\right] d x d y .
$$

Then, from (4.20) we deduce

$$
\begin{aligned}
\frac{d}{d t} F_{6}(t) \leq & -D \alpha\left[\left.\left\|\left.\psi_{t t}\right|_{H^{1}} ^{2}+\right\| \varphi_{t t}\right|_{H^{1}} ^{2}\right]+C \int_{\Omega}\left[\left|\psi_{t t t}\right|^{2}+\left|\varphi_{t t t}\right|^{2}\right] d x d y \\
& -K \int_{\Omega} \nabla w \cdot\left(\psi_{t t t t}, \varphi_{t t t t}\right) d x d y
\end{aligned}
$$

Finally we define

$$
F_{7}(t):=F_{5}(t)+\frac{C_{\beta}}{D \alpha} F_{6}(t)
$$

Then, from (4.19) and (4.22) we obtain

$$
\begin{aligned}
\frac{d}{d t} F_{7}(t) \leq \quad & -E_{1}(t)-\frac{\beta}{4}\|\nabla w\|_{L^{2}}^{2}+C \int_{\Omega}\left[\left|\psi_{t t t}\right|^{2}+\left|\varphi_{t t t}\right|^{2}\right] d x d y \\
& -K \int_{\Omega} \nabla w \cdot\left(\psi_{t t t t}, \varphi_{t t t t}\right) d x d y
\end{aligned}
$$

and we can deduce that

$$
\frac{d}{d t} F_{7}(t) \leq-E_{1}(t)+C_{0} \int_{\Omega}\left[\left|\psi_{t t t}\right|^{2}+\left|\varphi_{t t t}\right|^{2}+\left|\psi_{t t t t}\right|^{2}+\left|\varphi_{t t t t}\right|^{2}\right] d x d y,
$$

where $C_{0}>0$ is a constant defined as in (4.7), and also depends on the constants given by Korn's Inequality. 
Now we are in the position to prove the main result of this section.

Theorem 4.2. Suppose that the initial data verify

$$
U_{0}:=\left(w_{0}, w_{1}, \psi_{0}, \psi_{1}, \varphi_{0}, \varphi_{1}\right)^{\prime} \in D\left(\mathcal{A}^{4}\right) .
$$

Then the first order energy $E_{1}(t)$ associated to the system (1.11)-(1.13) with boundary conditions (2.1) decays polynomially to zero as time goes to infinity; that is, there exists a positive constant $C$, being independent of the initial data, such that

$$
E_{1}(t) \leq \frac{C}{t} \sum_{i=1}^{4} E_{i}(0)
$$

Moreover, if $U_{0} \in D\left(\mathcal{A}^{4 k}\right)$, then

$$
\left\|T(t) U_{0}\right\|_{\mathcal{H}} \leq \frac{C_{k}}{t^{k}}\left\|\mathcal{A}^{4 k} U_{0}\right\|_{\mathcal{H}}, \quad \forall k=1,2,3, \ldots,
$$

where $\{T(t)\}_{t \geq 0}$ is the semigroup associated to the system (1.11) -1.13) with infinitesimal generator $\mathcal{A}$ defined as in (2.3) .

Proof. We define $\mathcal{L}(t)$ as

$$
\mathcal{L}(t):=\frac{C_{0}}{d} \sum_{i=1}^{4} E_{i}(t)+F_{7}(t)
$$

where $d:=\min \left\{d_{1}, d_{2}\right\}>0$, with $d_{1}, d_{2}$ given by the system (1.11)-(1.13). Then, using (4.3) and (4.24) we obtain

$$
\frac{d}{d t} \mathcal{L}(t) \leq-E_{1}(t) .
$$

Therefore

$$
\int_{0}^{t} E_{1}(s) d s \leq \mathcal{L}(0)-\mathcal{L}(t), \quad \forall t \geq 0 .
$$

On the other hand, it is not difficult to prove that there exists a constant $C>0$ such that

$$
\mathcal{L}(0)-\mathcal{L}(t) \leq C \sum_{i=1}^{4} E_{i}(0), \quad \forall t \geq 0 .
$$

From (4.27)-(4.28) we obtain

$$
\int_{0}^{t} E_{1}(s) d s \leq C \sum_{i=1}^{4} E_{i}(0) .
$$

Then, since

from (4.29) we get

$$
\frac{d}{d t}\left\{t E_{1}(t)\right\}=E_{1}(t)+t \frac{d}{d t} E_{1}(t) \leq E_{1}(t),
$$

$$
E_{1}(t) \leq \frac{C}{t} \sum_{i=1}^{4} E_{i}(0),
$$

which completes (4.25) and shows that (4.26) holds, for $k=1$.

Finally, if $U_{0} \in D\left(\mathcal{A}^{4 k}\right) k \geq 2$, using that $0 \in \rho(\mathcal{A})$ (resolvent set of $\mathcal{A}$ ), we can apply [13. Proposition 3.1] to obtain (4.26), which completes the proof. 
Acknowledgements. The author should like to thank Professor Dr. R. Racke for his suggestions, corrections, and comments on this work.

\section{REFERENCES}

[1] Ammar Khodja, F., Benabdallah, A., Muñoz Rivera, J.E., Racke R.: Energy decay for Timoshenko systems of memory type. J. Differential Equations 194 (2003), 82-115. MR.2001030 (2004f:74032)

[2] Duvaut, G., Lions, J.L.: Inequalities in Mechanics and Physics. Springer-Verlag, Berlin. (1976). MR0521262 (58:25191)

[3] De Lima Santos, M.: Decay rates for solutions of a Timoshenko system with a memory condition at the boundary. Abstract and Applied Analysis 7(10) (2002), 531-546. MR 1932704 (2004e:74043)

[4] Fernández Sare, H.D., Muñoz Rivera, J.E.: Stability of Timoshenko systems with past history. J. Math. Anal. Appl. 339 (2008), 482-502.

[5] Fernández Sare, H.D., Racke, R.: On the stability of damped Timoshenko systems-Cattaneo versus Fourier law. Accepted for publication in Arch. Rat. Mech. Anal. (2008).

[6] Kim, J.U., Renardy, Y.: Boundary control of the Timoshenko beam. SIAM Journal of Control Optim. 25(6) (1987), 1417-1429. MR912448 (88m:93124)

[7] Lagnese, J.E.: Boundary Stabilization of Thin Plates. SIAM, Philadelphia (1989). MR1061153 (91k:73001)

[8] Lagnese, J.E., Lions, J.L.: Modelling, Analysis and Control of Thin Plates. Collection RMA, Masson, Paris, (1988). MR953313 (89k:73001)

[9] Liu, Z., Zheng, S.: Semigroups associated with dissipative systems. Research Notes Math. 398, Chapman\&Hall/CRC, Boca Raton (1999). MR1681343 (2000c:47080)

[10] Muñoz Rivera, J.E., Portillo Oquendo, H.: Asymptotic behavior on a Mindlin-Timoshenko plate with viscoelastic dissipation on the boundary. Funkcialaj Ekvacioj 46 (2003), 363-382. MR2035445 (2004k:74048)

[11] Muñoz Rivera, J.E., Racke, R.: Mildly dissipative nonlinear Timoshenko systems-global existence and exponential stability. J. Math. Anal. Appl. 276 (2002), 248-278. MR1944350 (2003i:35260)

[12] Muñoz Rivera, J.E., Racke, R.: Global stability for damped Timoshenko systems. Disc. Cont. Dyn. Sys. 9 (2003), 1625-1639. MR2017685 (2004j:35028)

[13] Prüss, J., Bátkai, A., Engel, K., Schnaubelt, R.: Polynomial stability of operator semigroups. Math. Nachr. 279 (2006), 1425-1440. MR2269247 (2007k:47067)

[14] Soufyane, A.: Stabilisation de la poutre de Timoshenko. C. R. Acad. Sci. Paris, Sér. I 328 (1999), 731-734. MR1680836 (2000b:74055) 